\section{AUDIT OF NEONATAL ADMISSIONS TO ACUTE PAEDIATRIC SERVICES AT ALDER HEY CHILDREN'S HOSPITAL WITH FEEDING PROBLEMS AMENABLE TO COMMUNITY-BASED SUPPORT}

Mary Masoud, Thomas Dunne, Kalvin Lee, Helen M Nabwera, Halina Kamarova. UK

10.1136/bmjpo-2021-RCPCH. 164

Background Alder Hey Children's Hospital has a tertiary acute general paediatric service with a very high number of admissions. In the context of reduced community-based support for mothers with young infants to establish and sustain breastfeeding or other appropriate infant feeding strategies, we were concerned that some of our neonatal admissions were inappropriately exposing infants to potentially life-threatening infections, whilst having an adverse impact on mothers and their families. We therefore conducted an audit to describe the population of neonates $<7$ days admitted to our acute general paediatrics services following discharge from maternity units in Merseyside, North West England. The aim was to identify potential targets of intervention that could avoid unnecessary admissions.

Objectives To evaluate causes of admission to acute general paediatric services for infants $<7$ days at Alder Hey Children's hospital to ascertain proportion with feeding problems amenable to community-based support.

Methods A retrospective audit of all infants less than 7 days of age admitted to Alder Hey Children's hospital, Liverpool from March to September 2019. All the infants were born and discharged from maternity units in Merseyside county, UK. Anonymised data was extracted from the electronic clinical records by three members of the clinical team. Implausible entries were discussed with all the audit team members at monthly meetings and excluded.

Results During the 6 months, there were 411 admissions, 160 (39\%) were surgical, and 251 (61\%) were medical. The majority of the medical admissions were to the acute general paediatric services 241(59\%). Of these 93 (23\%) had feeding problems. The majority were exclusively bottle -fed 42 (45.2\%), 35 (37.6\%) were exclusively breast fed and 16 $(17.2 \%)$ were mixed feeders. The majority had jaundice 41 $(44 \%)$ but only half of them required phototherapy (21, $22.6 \%)$ and a quarter $(23,24.7 \%)$ underwent a septic screen. However, 29 (31.2\%) presented solely with poor feeding.

The majority of infants with poor feeding didn't require any investigations or treatment $44(47.3 \%)$. The mean birth weight of $3.19 \mathrm{~kg}$ (SD 0.48), mean admission weight of $3 \mathrm{~kg}$ (SD 0.48) and mean discharge weight was $3.12 \mathrm{~kg}$ (SD 0.46). The mean difference between birth weight and admission weight was $7.3 \%$ and only $12(12.9 \%$ ) had lost $>10 \%$ birth weight at admission.

Conclusions Although feeding problems may be a sign of severe illness among infants, our audit showed that nearly a third of infants who were admitted in the first week of life to our acute tertiary general paediatric services, did not have any other features of severe illness. These admissions expose mothers and their infants to hospital acquired infections whilst disrupting the 'rooming-in' process that enhances the establishment of breastfeeding. These admissions could potentially be averted by optimising community infant feeding support services and discharge criteria from maternity care in the region. Engagement with these services will be a crucial next step in developing strategies to enhance infant feeding support particularly in impoverished communities in the region.

\section{PREGNANCY OUTCOMES IN WOMEN WITH CHRONIC KIDNEY DISEASE IN A SINGLE CENTER TERTIARY INSTITUTION}

Zhilin Kang, Mary Grace Tan, Woei Bing Poon. Singapore

10.1136/bmjpo-2021-RCPCH.165

Background It has been well known that mothers with chronic kidney disease (CKD), particularly end stage renal failure (ESRF), have high rates of infertility and pregnancyrelated complications such as pre-eclampsia, miscarriages, early preterm deliveries and small for gestational age (SGA) infants. Advances in multidisciplinary management including medical treatment and obstetric management of mothers with CKD in the past decade have greatly improved pregnancy outcomes. There is currently no recent data on outcomes of infants born to mothers with renal impairment in Singapore.

Objectives To stratify pregnancy outcomes of mothers with chronic kidney disease (CKD) by staging so as to improve neonatal counselling and prognostication.

Methods A single centre retrospective cohort study was conducted from August 2012 to December 2020 in a tertiary care centre in Singapore. The pregnancy outcomes of mothers with all CKD stages were included. Comparison was made between severe renal impairment (SRI) pregnancies with CKD stage 4 to 5 or requiring renal replacement therapy (RRT) versus those with mild to moderate renal impairment (MRI) in CKD stages 1 to 3 and not on RRT. Primary outcome was combined mortality or major morbidity including one or more of IVH, NEC, BPD and ROP. Secondary outcomes of interest were prematurity, small for gestational age (SGA), presence of non-reassuring fetal signs (NRFS) prior to delivery, requirement of extensive resuscitation, delivery via lower-segment cesarean section, whether the mothers had co-existing hypertension and use of anti-hypertensive during pregnancy. Discrete variables were analyzed using the chi square. Continuous variables were analyzed using Mann-Whitney $\mathrm{U}$ test. $\mathrm{P}<0.05$ was considered significant.

Results 74 pregnancies were included, of which 15 (20.2\%) were SRI pregnancies. Compared to MRI pregnancies, SRI pregnancies had significantly higher peak urea (median \pm interquartile range: $18 \pm 7.8$ versus $5.9 \pm 4.9 \mathrm{mmol} / \mathrm{L})$ and creatinine levels $(514 \pm 334$ versus $70 \pm 60 \mathrm{mmol} / \mathrm{L})$, infants had lower birthweight (1170 \pm 1185 grams versus $2475 \pm 1105$ grams) and gestational age (31 \pm 7 versus 36 \pm 4 ). Pregnancy with SRI was associated with more combined mortality or major morbidity (OR 5.889; 95\% CI 1.551-22.357), prematurity (OR 10.500; 95\% CI 1.27886.276), hypertension (OR 3.352, 95\% CI 1.039-10.817) and use of anti-hypertensive in pregnancy (OR 3.673, 95\% CI 1.131-11.936) as well as presence of NRFS (OR 7.125, 95\% CI 1.072-47.371). Sub-analyses done also showed greater odds of combined mortality and major morbidity if the CKD mothers

Conclusions Neonates of SRI pregnancies have significantly increased risk of combined mortality and morbidity, preterm birth, and foetal distress. Renal pregnancies with coexisting hypertension and especially those requiring anti-hypertensive medications had higher risks of combined mortality and morbidity. The importance of controlling hypertension and use of anti-hypertensives in CKD pregnancies are important for women with CKD who are attempting to conceive or who are already pregnant. 